\title{
Anti-Krebs-Diät
}

\section{Fischöl bremst PCa-Wachstum}

— Mit einer ungewöhnlichen Studie haben US-amerikanische Forscher nachgewiesen, dass die Ernährung das Teilungsverhalten

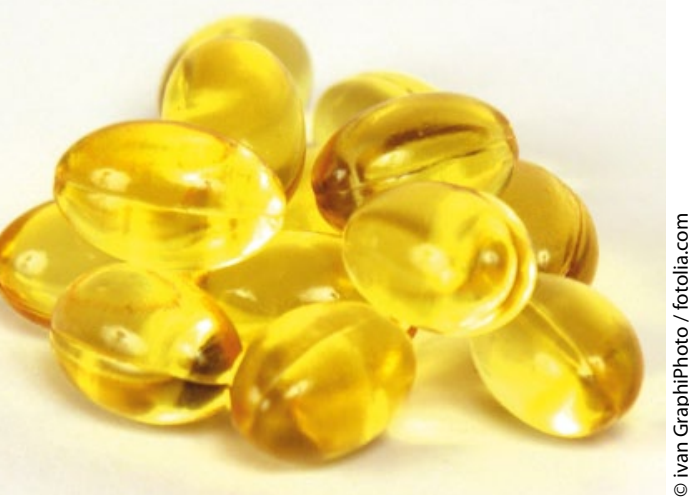

Eine Nahrungsergänzung mit Omega-3Fettsäuren hat offenbar einen positiven Effekt bei Prostatakrebs. von Prostata-Tumorzellen beeinflusst [Aronson WJ et al. Cancer Prev Res 2011; 4: 1-10]. In der Studie bekamen 55 Männer mit Prostatakarzinom vier bis sechs Wochen vor der Prostatektomie unterschiedliche Mahlzeiten aufgetischt: Etwa die Hälfte der Patienten bekam die übliche US-amerikanische Diät, bei der $40 \%$ der Kalorien überwiegend aus gesättigten Fettsäuren stammten, das Verhältnis von Omega-6- zu Omega-3-Fettsäuren betrug 15:1. Die andere Hälfte bekam eine fettreduzierte Diät, hier stammten nur $15 \%$ der Kalorien aus Fetten, und das Verhältnis von Omega-6- zu Omega-3-Fettsäuren lag bei 2:1. Um dieses günstige Verhältnis zu erreichen, erhielten die Teilnehmer in dieser Gruppe zusätzlich täglich $5 \mathrm{~g}$ Fischöl supplementiert. Die Mahlzeiten wurden von Klinikköchen zubereitet und den Männern zugestellt.
Nach der Prostatektomie nahmen die Forscher die Tumorzellen genauer unter die Lupe. Sie stellten fest, dass sich bei Männern mit der Fischöldiät das Fettsäureverhältnis in den Zellmembranen zugunsten von Omega-3-Fettsäuren verschoben hatte. Noch interessanter war jedoch das Teilungsverhalten der Zellen: Mit der Fischöldiät lag die Teilungsrate der Krebszellen ein Drittel unter der von Männern mit normaler Ernährung. Dies ist insofern relevant, als eine hohe Teilungsrate mit einer ungünstigen Prognose und einem erhöhten Metastasierungsrisiko einhergeht. Auch das Rezidivrisiko nach der Prostatektomie ist erhöht, wenn sich die Tumorzellen rasch teilen.

Über welche Faktoren der günstige Effekt vermittelt wird, ist noch unklar. Die Forscher hatten zunächst gehofft, mit der Fischöldiät die Konzentrationen des proliferationsfördernden Wachstumsfaktors IGF-1 im Blut zu verringern, dies war aber nicht der Fall. Sie vermuten daher andere Mechanismen.

Thomas Müller

\section{Kollateralschaden}

\section{Knockt die ESWL Spermien aus?}

— Werden bei Männern Harnsteine im unteren Abschnitt der Ureteren im Rahmen einer extrakorporalen Lithotripsie (ESWL) zertrümmert, bleibt das nicht ohne Auswirkung auf die Spermienqualität: Eine vorübergehende Oligoasthenozoospermie für etwa drei Monate ist meist Folge des Stoßwellenbeschusses, wie britische Wissenschaftler in einem aktuellen Review berichten [Gulur DM et al. BJU Int 2011; 108: 1321-3]. Die Forscher fanden vier Studien zu dieser Thematik, die alle zu ähnlichen Ergebnissen kamen. Untersucht hatte man jeweils Männer, die vor der Behandlung eine normale Spermaqualität und klinisch unauffällige Hoden hatten. Die Spermaqualität wurde ein Tag vor ESWL, ein bis sieben Tage danach sowie drei Monate später überprüft. Die Spermaqualität wurde gemäß der WHO-Leitlinie beurteilt.

Bei allen Probanden litt die Spermienqualität deutlich unter der ESWL. Die Spermienzahl und -motilität sank nach der Behandlung im Vergleich zu vorher signifikant, und das Sperma enthielt teils schon makroskopisch erkennbare, teils erst unter dem Mikroskop detektierbare Blutmengen. Ein Autorenteam wies sogar deutlich mehr missgebildete Spermien nach. Bei der Frage, ob es sich hier um eine bleibende oder vorübergehende Schädigung handelt, ist man sich nicht so einig: In drei Studien erholte sich die Spermienqualität drei Monate nach ESWL wieder und war ähnlich gut wie vor der Behandlung, in einer blieb sie deutlich darunter.

Die Autoren folgern, dass eine ESWL zumindest vorübergehend die Spermaqualität verschlechtert. Zwar handele es sich um eine zeitlich begrenzte Beeinträchtigung, doch um eine klare Entwarnung aussprechen zu können, bedürfe es größerer Studien mit einem längeren Follow-up. Ganz unklar bliebe, welche Konsequenz eine ESWL für Männer habe, deren Spermaqualität schon vorher suboptimal war.

Dr. Dagmar Kraus
KBV dreht den Spieß um

"Kassen-Navigator" in den Startlöchern

- Das Internet wird zur Arena. Als Reaktion auf die Arztbewertungsportale der Krankenkassen bereitet die Kassenärztliche Bundesvereinigung (KBV) einen „Kassen-Navigator“ vor. KBV-Chef Dr. Andreas Köhler kündigte an, das Bewertungsportal im Laufe des neuen Jahres an den Start zu bringen. Wenn die Kassen ihren eigenen Ansprüchen, die Versicherten ausreichend zu informieren, nicht genügten, dann müsse die Ärzteschaft dies selbst in die Hand nehmen, betonte Köhler. Versicherte könnten damit im Internet das Leistungsangebot ihrer Kassen bewerten. Damit reagiert die KBV auf die Arztbewertungsportale, die die AOK, die Barmer GEK und der Verband der Ersatzkassen in der jüngeren Vergangenheit aufgelegt haben. Bislang haben $10 \%$ der Versicherten, die an einer Umfrage der Forschungsgruppe Wahlen teilgenommen haben, ein solches Portal genutzt. Von diesen hat laut der Umfrage mehr als die Hälfte die Arztbewertungen im Internet als nicht hilfreich empfunden, Tendenz steigend. „Die Krankenkassen überschätzen die Relevanz von Arztbewertungsportalen", betonte KBVVorstand Dr. Carl-Heinz Müller dazu.

Anno Fricke 